\title{
La restauration des films d'Albert Capellani détenus par les Archives françaises du film (CNC)
}

\author{
Caroline Patte
}

\section{CpenEdition}

\section{Journals}

Édition électronique

URL : http://journals.openedition.org/1895/4743

DOI : $10.4000 / 1895.4743$

ISSN : 1960-6176

\section{Éditeur}

Association française de recherche sur l'histoire du cinéma (AFRHC)

\section{Édition imprimée}

Date de publication : 1 décembre 2012

Pagination : 235-240

ISBN : 978-2-913758-63-6

ISSN : 0769-0959

Référence électronique

Caroline Patte, « La restauration des films d'Albert Capellani détenus par les Archives françaises du film (CNC) », 1895. Mille huit cent quatre-vingt-quinze [En ligne], 68 | 2012, mis en ligne le 01 décembre 2015, consulté le 23 septembre 2019. URL : http://journals.openedition.org/1895/4743 ; DOI : $10.4000 / 1895.4743$ 


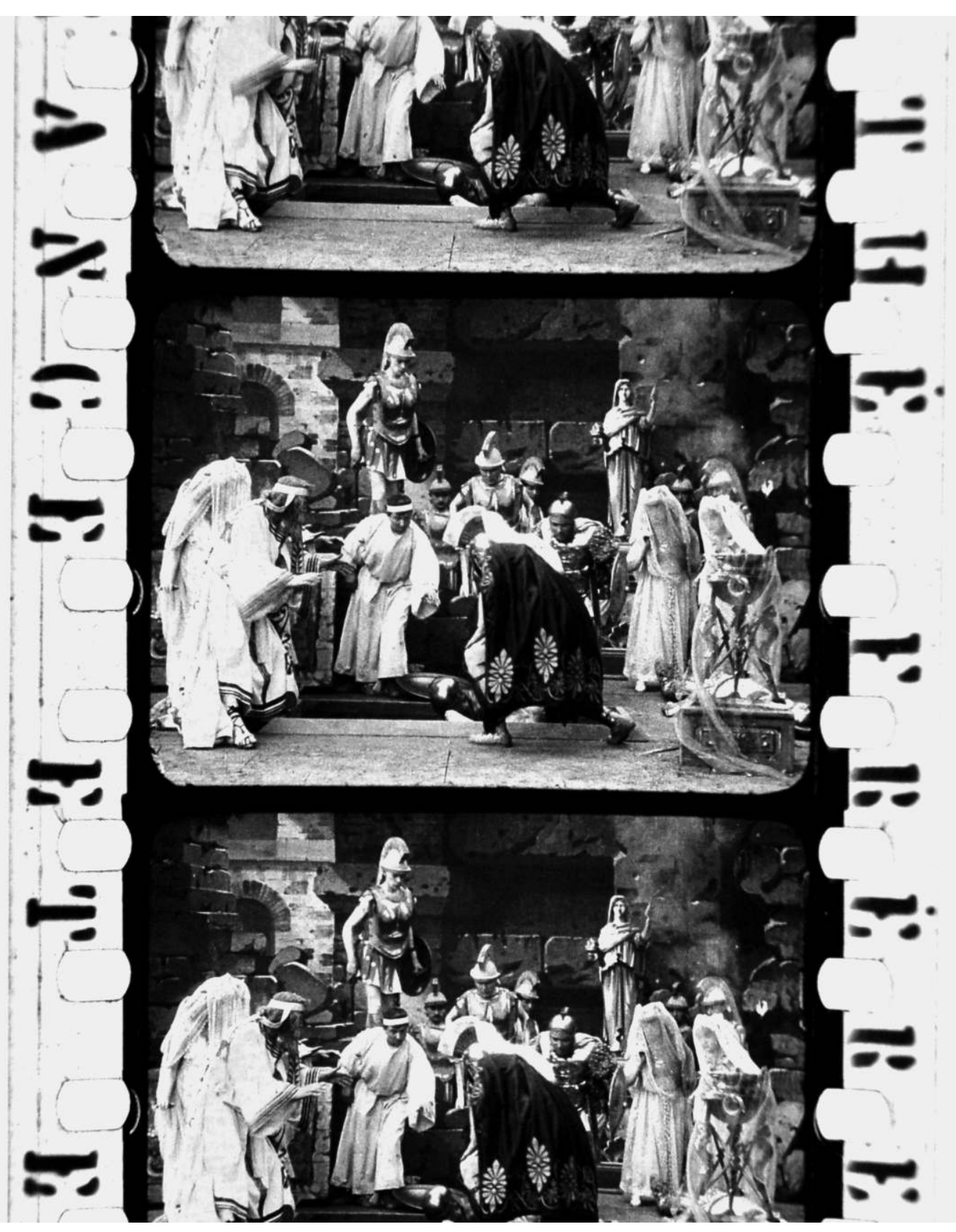

La Vestale: copie d'origine.

๙্ণ 


\section{La restauration des films d'Albert Capellani détenus par les Archives françaises du film ( $\mathrm{CNC}$ )}

Caroline Patte

Avec la création des Archives françaises du film du Centre national du cinéma et de l'image animées (CNC) en 1969, l'Etat prenait avant tout en charge l'inventaire et la conservation des films anciens sur support nitrate. Mais l'objectif était aussi d'entreprendre des travaux de restauration pour les éléments particulièrement en danger. Avec la mise en place d'un laboratoire autonome, au début des années 70, puis avec le lancement du "plan nitrate» au début des années 90, un travail de restauration d'envergure s'est mis en place. Dès les premières années d'activité des Archives françaises du film, Albert Capellani a fait l'objet d'un intérêt particulier, avec entre autre la publication des recherches de Charles Ford sur la carrière du réalisateur et avec la prise en charge de la restauration de ses films déposés aux Archives françaises du film (AFF) ${ }^{1}$.

Le travail d'inventaire et d'identification est un préalable nécessaire avant tous travaux de restauration. L'analyse et la comparaison de tout le matériel lié à un film permet de sélectionner les meilleurs éléments pour effectuer la restauration. Cette sélection se fait selon des critères physiques (état de la pellicule, longueur ou durée, état des couleurs) et techniques (nature de l'élément). Un négatif sera toujours le meilleur élément d'un point de vue technique, mais pas toujours le plus intéressant.

La collection de films d'Albert Capellani conservée aux AFF provient de différents dépôts. Il s'agit dans la plupart des cas de copies d'exploitation, parfois d'éléments uniques, avec ou sans intertitres, souvent en couleurs et dans un état physique variable. Nous avons pu constater des dégradations physiques, biologiques ou chimiques liés à la nature du support de la pellicule et des conditions de conservation. Dans le cas de négatifs, nous sommes obligatoirement en présence d'éléments noir et blanc, non montés dans l'ordre. Les plans sont assemblés en rouleaux selon la lumière nécessaire au tirage et selon la teinte finale dans la copie. Les rouleaux comprennent aussi les indications d'emplacement des intertitres.

Très tôt, la couleur est apparue au cinéma en utilisant divers procédés. Les principales techniques utilisées étaient le coloriage et le pochoir ou encore le teintage. Elles s'appliquaient sur un positif noir et blanc. Dans le cas des coloriages, l'application de plusieurs couleurs se faisaient aux pinceaux de

1. Le CNC a édité en 1984 la monographie de Charles Ford Albert Capellani, précuseur méconnu. Malgré ses imperfections et ses erreurs, cette publication unique à l'époque marque l'intérêt de l'Institution pour l'œuvre du cinéaste, mais curieusement sans s'appuyer sur les collections déposées au CNC. Or, plusieurs titres étaient déjà présents, déposés par des collectionneurs pour la plupart. Certains travaux ont été réalisés il y a plusieurs années, mais le projet de programmation du festival Il Cinema ritrovato a placé le travail de restauration de l'œuvre de Capellani au cœur des activités du $\mathrm{CNC}$, vingt ans après la publication de l'ouvrage de Ford. 
manière manuelle sur chaque image. La technique du pochoir reprend le principe du coloriage, mais à travers des pochoirs. Dans le cas du teintage, la mise en couleur se faisait par des passages dans des bains colorés. Les solutions colorées se déposaient sur le support de la pellicule. Chaque couleur de teintage étant bien souvent choisie en fonction des ambiances ou de repères spatiaux. Les indications de couleurs étaient notées sur les amorces qui précédaient chaque rouleau de tirage. C'est après le tirage des éléments positifs qu'avait lieu le montage narratif avec insertion des cartons titres en français ou dans une autre langue selon le pays de diffusion.

Quand nous n'avons que le négatif entre les mains, il est difficile de restituer les couleurs d'origine souhaitées, sans références visuelles. Sauf dans le cas des films Pathé, il est possible de se baser sur les références codifiées des couleurs dans l'ouvrage Le Film vierge Pathé.

Jusque dans les années 70, la restauration des films couleur muets se faisait beaucoup en noir et blanc. C'est pourquoi, les spectateurs ont oublié que ces films pouvaient être projetés en couleur. Par la suite, les Archives et Cinémathèques, dont les AFF, ont entrepris à nouveau des restaurations en couleurs de ces films à partir de références solides. A présent, les spectateurs peuvent redécouvrir la couleur sur ces films muets.

Dans le cas des films d'Albert Capellani, faute de négatifs, nous avions donc essentiellement en dépôt des copies couleurs. De cette façon, la restauration entreprise permit la restitution des couleurs de l'original, du moins telles qu'elles nous sont parvenues. De plus, les copies possédaient aussi leurs intertitres d'origine. La restauration se fait en dupliquant les copies sur un support intermédiaire couleur. Avec ce procédé, les couleurs obtenues sont proches des couleurs originales, mais le film conserve certains défauts laissés par le passage du temps (décomposition, fadeur, etc.). Même si ce procédé peut faire perdre du contraste, nous sommes au plus proche des couleurs de l'original.

Dans le cas de ce corpus de films de Capellani identifiés et restaurés aux Archives françaises du film, il y a autant de situations qu'il y a de films. Chaque restauration a été un défi particulier. Et c'est avec beaucoup d'enthousiasme que ce travail a été entrepris en prévision de la rediffusion des films auprès d'historiens, de chercheurs, et aussi du public, revisitant l'œuvre du cinéaste pour la première fois depuis des décennies. L’ensemble des travaux menés dans les différentes Archives et Cinémathèques a permis de réévaluer l'apport de Capellani à l'histoire du cinéma, d'étudier les techniques utilisées par les laboratoires d'origines (Pathé), et de mieux percevoir la place centrale du cinéaste dans les premières années du cinéma français. À noter que l'inventaire des collections se poursuit, avec l'espoir de retrouver encore des films considérés comme perdus d'Albert Capellani. 
Fiches techniques de restauration des films d'Albert Capellani

détenus par les Archives françaises du film.

La Loi du pardon (1906)

Elément d'origine: Copie $35 \mathrm{~mm}$ nitrate, déposée par un collectionneur. $138 \mathrm{~m}, 7$ minutes, $16 \mathrm{i} / \mathrm{s}$. noir et blanc + intertitres teintés en rouge. État médiocre. Léger affaiblissement de l'image par intermittence. Image légèrement affecté de taches bleues. Absence carton mot fin.

Date dépôt : 1978

Elément obtenu: copie $35 \mathrm{~mm}, 137 \mathrm{~m}, 7$ minutes, 16 $\mathrm{i} / \mathrm{s}$. avec reconstitution des intertitres.

Date des travaux: 1994.

Note: Restauration à partir d'une copie nitrate noir et blanc mais qui présente des défauts photographiques avec affaiblissement de l'image par intermittence. Mais la restauration a permis de rattraper ces défauts.

\section{La Fille du sonneur (1906)}

Elément d'origine: Copie $35 \mathrm{~mm}$ nitrate acquise par le CNC auprès d'un collectionneur. $194 \mathrm{~m}, 10$ minutes, $16 \mathrm{i} / \mathrm{s}$, noir et blanc + teinté. Titre et intertitres trop courts. Défauts physiques majeurs: décomposition et perforations arrachées par intermittence.

Date dépôt : 1981

Elément obtenu: copie $35 \mathrm{~mm}, 197 \mathrm{~m}, 10$ minutes, 16i/s, noir et blanc + teinté. Avec des passages de décomposition photographique. Remise à longueur du titre et des intertitres pour une meilleure lecture.

Date des travaux: 1993.

Note: La copie a été acquise par le CNC en 1981, et elle est la seule copie existante connue. Elle présentait des signes de décomposition en début sur environ 40 mètres. Par conséquent la copie obtenue en couleur est incomplète avec de nombreux défauts photographiques dus à l'état physique de l'original. Malgré cela, la restauration s'est faite en couleur avec remise à longueur de lecture des intertitres et du titre. Malgré le manque de début, la narration reste compréhensible.

Cendrillon ou la pantoufle merveilleuse (1907)

Elément d'origine: Copie $35 \mathrm{~mm}$ nitrate, $260 \mathrm{~m}$, 14 minutes, $16 \mathrm{i} / \mathrm{s}$, noir et blanc + teinté + pochoir (sur les 16 derniers mètres). Il manque le début et le mot

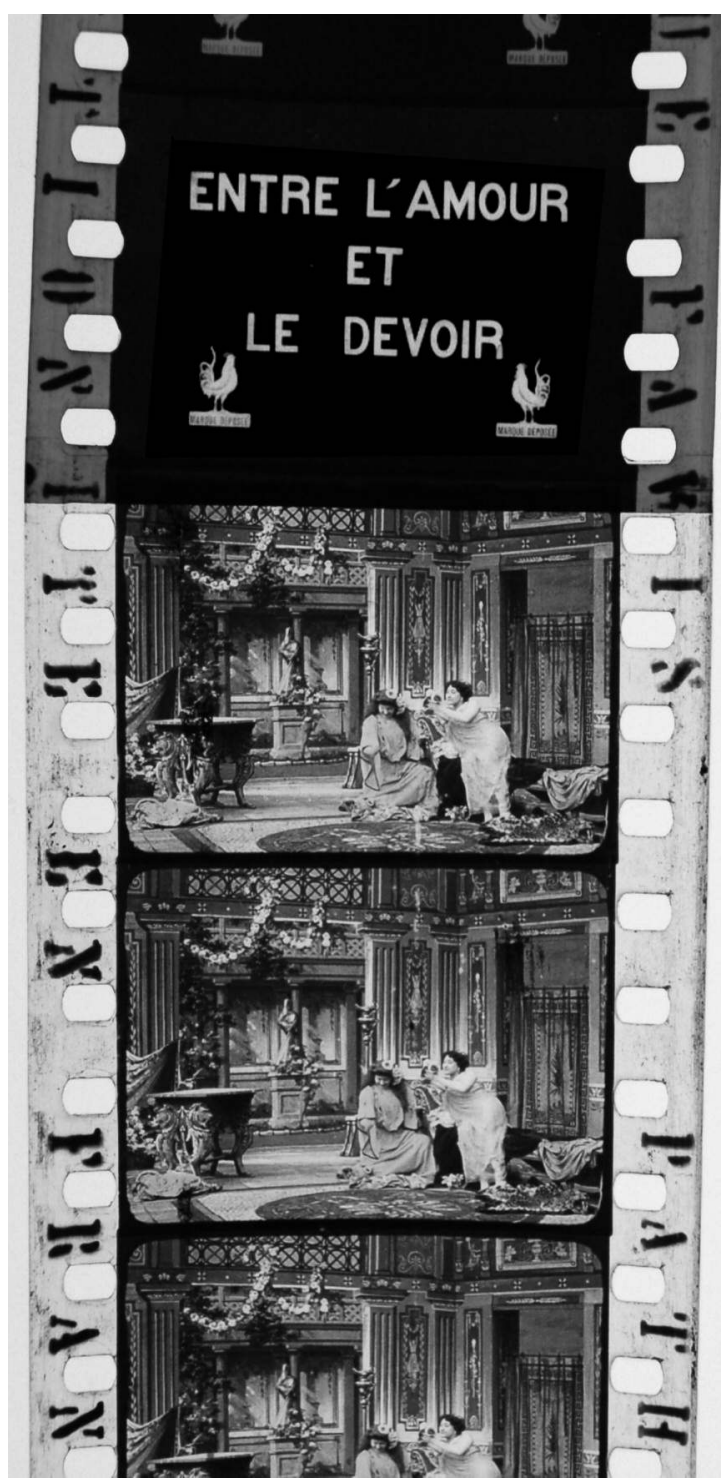

La Vestale: copie d'origine avec intertitre Pathé. 
fin. Intertitres en anglais. Elle présente de nombreuses rayures côté émulsion et côté support.

Cette copie a été donnée aux Archives françaises du film du CNC par la Bundesarchiv en 1988 dans le cadre des échanges entre membres de la Fédération Internationale des Archives du Film (FIAF).

Date dépôt : 1988

Elément obtenu: une copie $35 \mathrm{~mm} 269 \mathrm{~m}, 13$ minutes, $16 \mathrm{i} / \mathrm{s}$, couleur; remontage d'intertitres français issus d'une copie appartenant à une institution privée française, mais présentant des couleurs dénaturées et de nombreux défauts physiques, et plus incomplète (noir et blanc + teinté + pochoir, $252 \mathrm{~m}$ )

Date des travaux: 1994

Note: Malgré l'absence de début et de fin, la copie de la Bundesarchiv présente une continuité narrative.

\section{Les Deux Sours (1907)}

Elément d'origine: Copie $35 \mathrm{~mm}$ nitrate, déposée par un collectionneur. $194 \mathrm{~m}, 9$ minutes, $16 \mathrm{i} / \mathrm{s}$. Noir et blanc + teinté. Avec de nombreux défauts physiques dont des tâches de rouille en fin.

Il existe aussi un négatif nitrate en 8 rouleaux non montés, moisi, pellicule Pathé à une perforation avec indication des emplacements des intertitres.

Date dépôt : 1980

Elément obtenu: copie $35 \mathrm{~mm}, 194 \mathrm{~m}, 9$ minutes, $16 \mathrm{i} / \mathrm{s}$, noir et blanc + couleur.

Date des travaux: 1992

Note: Les tâches de moisi n'ont pu être complètement éliminées lors de la restauration.

\section{Les Apprentissages de Boireau (1907)}

Elément d'origine: Copie nitrate incomplète, $35 \mathrm{~mm}$, $108 \mathrm{~m}, 5$ minutes, $16 \mathrm{i} / \mathrm{s}$. Noir et blanc + teinté, intertitres en anglais. Il manque environ $100 \mathrm{~m}$ en fin.

Cette copie a été donnée aux Archives françaises du film du CNC par la Bundesarchiv en 1988 dans le cadre des échanges entre membres de la Fédération Internationale des Archives du Film (FIAF).

Date de dépôt: 1988

Elément obtenu: Copie $35 \mathrm{~mm}, 105 \mathrm{~m}, 6$ minutes, $16 \mathrm{i} / \mathrm{s}$, couleur. Incomplète. Manque de fixité dû à l'original.

Date des travaux : 1996

Note: Récemment, une nouvelle copie plus complète a été déposée aux AFF. Une nouvelle restauration pourrait être envisagée prochainement.

\section{La Légende de Polichinelle (1907)}

Elément d'origine: copie $35 \mathrm{~mm}$ nitrate déposée par une institution privée. $245 \mathrm{~m}, 13$ minutes, 16 i/s, noir et blanc. Défaut physique majeur: gélatine fondue.

Date de dépôt: 1990

Elément obtenu: contretype $35 \mathrm{~mm}, 212 \mathrm{~m}$, 11 minutes, $16 \mathrm{i} / \mathrm{s}$. noir et blanc. Incomplet. Image affaiblie sur la moitié du film.

Date des travaux: 1991

Note: Travaux de sauvegarde d'un élément en mauvais état, alors qu'à l'époque le film était dit perdu.

\section{Aladin ou la Lampe merveilleuse (1907)}

Elément d'origine: copie $35 \mathrm{~mm}$ nitrate déposée par une institution d'Etat. $225 \mathrm{~m}, 10$ minutes, 16 i/s, noir et blanc + pochoir + teinté. Avec des traces de rouille et une absence partielle d'image.

Date de dépôt : 1972

Elément obtenu: Internégatif $35 \mathrm{~mm}, 236 \mathrm{~m}$, 12 minutes, $16 \mathrm{i} / \mathrm{s}$. Noir et blanc + couleur.

Date de la sauvegarde: 1974

Note: Travaux de sauvegarde d'un élément en mauvais état, alors qu’à l'époque le film était dit perdu.

\section{Le Pied de mouton (1907)}

Elément d'origine: copie $35 \mathrm{~mm}$ nitrate, déposée par un collectionneur. $278 \mathrm{~m}, 15$ minutes, $16 \mathrm{i} / \mathrm{s}$, noir et blanc + pochoir (Procédé Pathécolor) + intertitres teintés

Date dépôt: 1974

Elément obtenu: copie $35 \mathrm{~mm}, 282 \mathrm{~m}, 15$ minutes, 16 i/s. Couleur. Quelques sauts dans l'image et manque de fixité sur quelques séquences.

Date des travaux: 1994

Note: Le tirage d'une nouvelle copie en 2000 a été nécessaire, pour répondre aux nombreuses demandes de projection. Création d'un carton de présentation du titre, pose du mot fin.

\section{La Vestale (1908)}

Elément d'origine: copie $35 \mathrm{~mm}$ nitrate, déposée par un collectionneur. $206 \mathrm{~m}, 11$ minutes, $16 \mathrm{i} / \mathrm{s}$, noir et blanc + teinté + pochoir (Procédé Pathécolor). Absence du mot fin.

Date dépôt: 1974

Elément obtenu: copie $35 \mathrm{~mm}$ inversible couleur, $212 \mathrm{~m}, 11 \mathrm{minutes}, 16 \mathrm{i} / \mathrm{s}$, cadrage sonore.

Date des travaux: 1995 
Note: restauration complète de l'élément, avec pose d'un carton de présentation du titre. Le choix d'une copie inversible s'est imposé par souci d'économie.

\section{Le Chat botté (1908)}

Elément d'origine: copie $35 \mathrm{~mm}$ nitrate incomplète, déposée par un collectionneur. $200 \mathrm{~m}, 11$ minutes, $16 \mathrm{i} / \mathrm{s}$. Noir et blanc + pochoir, en très mauvais état. Défaut physique majeur : gélatine fondue par intermittence.

Date dépôt : 1974

Elément obtenu : copie 35 mm, $202 \mathrm{~m}, 11$ minutes, 16 $\mathrm{i} / \mathrm{s}$, noir et blanc, incomplet.

Date des travaux: 1976

Note: Restauration à partir d'un élément au pochoir en très mauvais état. Les couleurs étaient trop dénaturées pour envisager une restauration couleur.

Notre-Dame de Paris (1911)

Elément d'origine: copie $35 \mathrm{~mm}$ nitrate en 3 bobines déposée par une institution privée. 719 m, 39 minutes, $16 \mathrm{i} / \mathrm{s}$. Noir et blanc + intertitres teintés. Pellicule gondolée par intermittence. Mot fin trop court.

Date dépôt : 1984

Elément obtenu: copie $35 \mathrm{~mm}, 725 \mathrm{~m}, 39$ minutes, $16 \mathrm{i} / \mathrm{s}$, noir et blanc. (intertitres noir et blanc)

Date des travaux: 1995

Note: Seuls les intertitres étaient en couleur (orange et vert). C'est pourquoi le choix a été fait de faire une restauration uniquement en noir et blanc de tout le film. Remise à longueur de lecture du mot fin.

\section{La Belle Limonadière (1914)}

Elément d'origine: négatif image avec parfois des plan en tri-acétate, déposé par une société de production. $1425 \mathrm{~m}, 69 \mathrm{~min}, 18 \mathrm{i} / \mathrm{s}$. défauts de dépolissage. Non montés: indication de l'emplacement des intertitres par des croix.

Date de dépôt: 2001

Elément obtenu: Copie 35 mm, 1432 m, 69 m, 18i/s. sans intertitres. Traces de moisissures par intermittence, élément issu de supports différents. Effet de pompage Date des travaux: 2001
Note: Travaux incomplets car il manque les intertitres. La narration n'est pas compréhensible.

\section{Social hypocrites (1918)}

Elément d'origine: copie $35 \mathrm{~mm}$ nitrate déposée par une institution d'Etat. $1054 \mathrm{~m}, 51$ minutes, $18 \mathrm{i} / \mathrm{s}$. noir et blanc + teinté

Date de dépôt: 1971

Elément obtenu: copie $35 \mathrm{~mm}, 1.140 \mathrm{~m}, 18 \mathrm{i} / \mathrm{s}$. noir et blanc.

Date des travaux: 2012.

Note: Le contretype date de 1974. Les intertitres présentaient un problème de contraste important. La copie a été tirée en 2012.

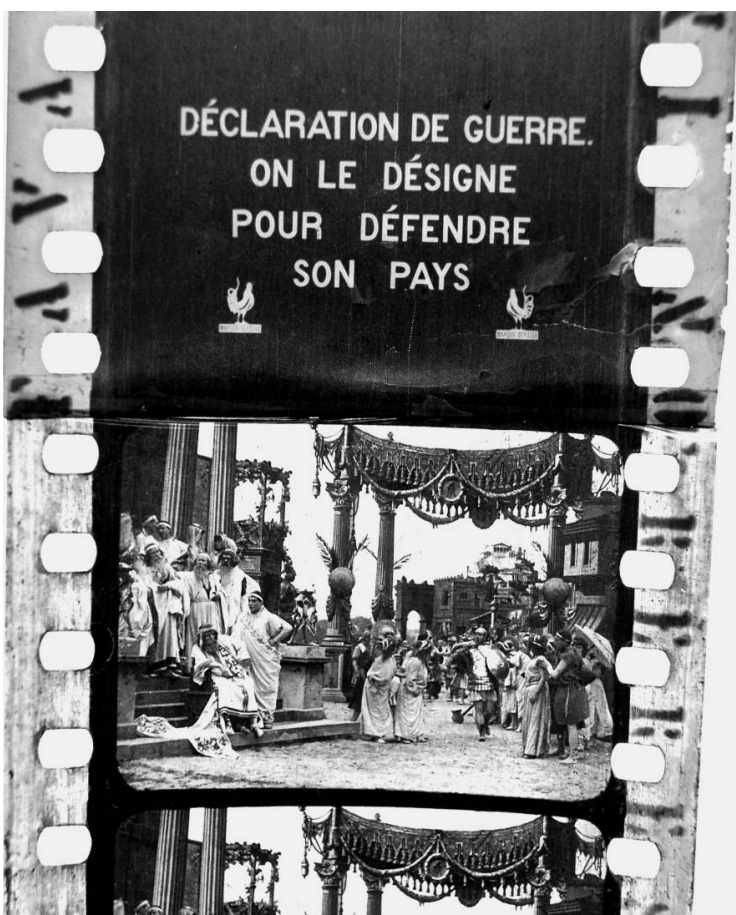

La Vestale: copie d'origine avec intertitre Pathé. 